\title{
Einsatz von Qualitätsindikatoren aus Routinedaten aus Sicht der BQS
}

Das GKV-Wettbewerbsstärkungsgesetz (GKVWSG) ermöglicht die Einbeziehung sowohl von medizinischen Routinedaten nach dem bisherigen BQS-Verfahren als auch künftig den Rückgriff auf administrative Routinedaten in die einrichtungs- und sektorenübergreifende Qualitätssicherung. Beide Instrumente haben spezielle Vor- und Nachteile. Sie sollten künftig in differenzierter Weise kombiniert werden. Wann immer bereits vorhandene Daten zur Qualitätsmessung zur Verfügung stehen, sollten sie genutzt werden, sofern dies rechtlich verbindlich und datenschutzrechtlich möglich ist.

Mit administrativen Routinedaten kann ein Screeningverfahren etabliert werden, das ohne weitere Zusatzdokumentation eine grob gerasterte Qualitätsdarlegung etlicher neuer Versorgungsbereiche ermöglicht. Bei Auffälligkeiten, die sich mit administrativen Routinedaten nicht mehr detailliert genug abbilden lassen, würde man dann problemfokussiert auf gesondert zu dokumentierende medizinische Daten zurückgreifen, um Verbesserungspotentiale erkennen zu können. Durch Differenzierungen der Diagnose- und Prozeduren-Klassifikationen könnten zukünftig wichtige Aspekte der Qualitätsdarlegung bereits durch administrative Routinedaten abgebildet und der Dokumentationsaufwand weiter reduziert werden.

Autorenerklärung: Es bestehen keine finanziellen Interessenkonflikte in Zusammenhang mit diesem Artikel.
C. Veit

Qualitätsmanagement

Schlüsselwörter

Qualitätssicherung

Routinedaten

Screening

Key words

quality improvement

administrative data

screening

Institut

BQS Bundesgeschäftsstelle Qualitätssicherung gGmbH, Düsseldorf

Bibliografie

DOI 10.1055/s-0028-1085584 Dtsch Med Wochenschr 2008; 133: S137 . @ Georg Thieme Verlag KG Stuttgart · New York . ISSN 0012-0472

Korrespondenz Dr. med. Christof Veit, Geschäftsführer BQS Bundesgeschäftsstelle Qualitätssicherung gGmbH Kanzlerstr. 4 D-40472 Düsseldorf Tel. +49 (0)211/280729-0 $\mathrm{Fax}+49$ (0)211/280729-99 eMail christof.veit@bqs-online.dewww.bqs-online.de 